\title{
PRECARIETÀ DEL LAVORO E CRISI DELLE MODERNE FORME DI CITTADINANZA SOCIALE
}

https://doi.org/10.17979/relaso.2011.1.1.1198

ENZO NOCIFORA

SAPIENZA, UNIVERSITA DI ROMA

\section{RESUMEN}

En todas las economías avanzadas del planeta, estamos hablando de un solo tema: cómo detener la crisis financiera destructiva y reiniciar el ciclo de crecimiento económico. Nuestra propuesta es volver a considerar la cultura de trabajo significativo para explicar la estructura de la sociedad. Esto es porque el trabajo es la única actividad humana que da sentido a la existencia. En los últimos dos siglos los trabajadores para fortalecer sus derechos de ciudadanía se han discutido con el Estado-nación en forma histórica que se ha consolidado durante las revoluciones liberales del siglo XIX. Hoy en día este es el vínculo que ha entrado en una crisis irreversible y es incapaz de proporcionar resultados que son adecuados para las emergencias. Nacieron organismos supranacionales que ciertamente no son iguales a sus tareas, y las fuerzas sociales no pueden encontrar una manera de contribuir a su empoderamiento político. Pera no nació un movimiento europeo de los trabajadores, que lucha por nacer en la misma forma que una Europa política capaz de detener el creciente poder de la especulación financiera. Los mercados financieros están integrados globalmente, mientras que los empleados, como en el pasado, presente sus solicitudes a escala nacional

Esta es la pregunta que ahora se ha convertido en urgente. Si queremos salir de la crisis económica, debemos pensar en términos de supra-nacional y europeo. Es importante que el movimiento de los trabajadores imponga sus propias exigencias a escala europea, a favor del fortalecimiento político de la Unión, y colocar allí el tema de los derechos sociales europeos. Sólo un movimiento obrero que trabaja para fortalecer la construcción de Europa, en lugar de debilitar abre los derechos de ciudadanía de los trabajadores

Revista Latina de Sociología, $\mathrm{n}^{\circ}$ 1: 126-155

http://revistalatinadesociologia.com ISSN 2253-6469 
PALABRAS CLAVE: cultura del trabajo, crisis económica, globalización de los mercados, derechos de los trabajadores, politica social

\section{ABSTRACT}

In all advanced economies of the planet, right now you're talking about a single issue: how to stop the destructive financial crisis and restart the cycle of economic growth. Our proposal is to go back to seeing the work culture significant for explaining the structure of the society. This is because labor is the only human activity that gives meaning to existence.

Over the last two centuries the workers to strengthen their rights of citizenship have discussed with the nation-state in historical form that has been consolidated during the nineteenth century liberal revolutions. Today this is the bond that has entered into an irreversible crisis and is unable to provide results that are adequate to emergencies. They were born supra-national bodies that are certainly not equal to their tasks, and social forces can not find a way to contribute to their political empowerment. So as not born a European movement of workers, struggling to be born in the same way a political Europe capable of stopping the growing power of financial speculation. Financial markets are integrated globally while the employed, as in the past, present their requests on a national scale.

This is the question now has become urgent. If we want to get out of the economic crisis we must think in terms of supra-national and European. It is important that the movement of workers imposed its own demands on a European scale, becoming in favor of strengthening the Union's political, and placing the issue of the European social rights. Only a labor movement that works to strengthen the construction of Europe, rather than opens weakening, the citizenship rights of workers. 
KEYWORDS: Work culture, Economic crisis, Globalization of markets, Workers' rights, Social policy

\section{Premessa}

I caratteri originari della crisi economica e sociale che stiamo vivendo non sono affatto chiari e definiti come potrebbe apparire ad un'analisi sommaria delle forze in campo. La centralità delle questioni poste dai mercati finanziari globalizzati, a cominciare dal rientro di un debito pubblico nazionale che si rifinanziava di anno in anno senza pericolo alcuno da almeno venti anni a questa parte, pongono con chiarezza la questione della sovranità nazionale e del pericolo che corrono i diritti sociali di cittadinanza in un quadro storico che è gravemente ipotecato da potentati sovranazionali che non hanno legittimazione alcuna.

Se si vuole anche solo rispondere a tono ai ricatti dei mercati e allo strapotere delle agenzie di rating occorre con chiarezza uscire dalla logica della finanza internazionale e rovesciare l'ordine delle questioni. In altri termini porre con fermezza i temi che sono veramente centrali per il paese, dal punto di vista dei lavoratori italiani. Riaffermare la centralità, sociale e politica, del lavoro vuol dire in sostanza rilanciare il tema della cittadinanza sociale in continuità con le conquiste di quel "secolo socialdemocratico"1 che sono state il carburante decisivo dello sviluppo europeo di tutto il Novecento.

D'altro canto è abbastanza intuitivo constatare che il rientro del debito è possibile solo in un clima di cocoperazione sociale e di redistribuzione egualitaristica di vantaggi e svantaggi, di benefici

1 La definizione è di un teorico autenticamente liberale come Ralf Dahrendorf che ci ha insegnato molte più cose di tanti sedicenti riformisti, socialdemocratici a parole, ma reazionari nei fatti.

Cfr. R. Dahrendorf, Libertà attiva : sei lezioni su un mondo instabile, Laterza, Bari 2005; Darhendorf R., La liberta che cambia, Laterza, Bari 1981. 
e vincoli, non in un clima di scontro e di intensificazione degli squilibri e delle disuguaglianze. Se si rompe il patto sociale fra i diversi settori della società il rientro del debito sarà semplicemente impossibile, ed i costi di questa rottura, alla fine della musica, li pagherà il paese nel suo complesso e, quindi, anche settori sociali oggi dominanti e di solito privilegiati.

Uscire dalla crisi è possibile ma solo se si innesca un circuito virtuoso di sostanziale crescita dei diritti dei lavoratori. Questa è, in estrema sintesi la tesi che ci ripromettiamo di dimostrare con questo breve saggio, che ha carattere del tutto introduttivo, rispetto ad una problematica complessa e articolata che potremo analizzare esaustivamente solo in altra sede.

\section{Lavoro e rappporti sociali di produzione}

La questione che più di ogni altra ha legato le mani al movimento operaio e sindacale italiano, nel corso degli anni Settanta, è connessa con la teoria della cosiddetta "fine del lavoro" 2 . E' nella seconda metà degli anni Settanta che i teorici della "società postindustriale"3, teorizzanno a dire il vero, non tanto la fine del lavoro in quanto tale, ma la fine della centralità del lavoro, in altri termini l'impossibilità di interpretare la società a partire dalla categoria del lavoro industriale moderno.

Si trattava di un ragionamento complesso che non era privo di qualche fondamento. Da un lato vi era infatti la progressiva riduzione della durata della giornata lavorativa e del crescente

2 Per una sintesi di questo tipo di posizione basti citare lo stupefacente testo di J. Rifkin la cui nonchalance teorica lascia del tutto sbalorditi. Ma d'altro canto finchè ci sono prestigiose università italiane che pagano salato per ospitare le sue conferenze è del tutto comprensibile il carattere di avventurosa e titatnica improvvisazione del personaggio.

Cfr. J. Rifkin, La fine del lavoro. Il declino della forza lavoro globale $e$ l'avvento dell'era post-mercato, Baldini \& Castoldi, Milano 2001.

3 Cfr. D. Bell, Appunti sulla società post-industriale, Torino 1967; A. Touraine, La società post-industriale, Il Mulino, Bologna 1974, D. De Masi (a cura di), L'avvento post-industriale, F. Angeli, Milano 1985. 
spazio sociale del tempo di non-lavoro, che appariva in quella fase come un processo strutturale inevitabile, che avrebbe presto rotto la barriera delle quaranta ore di lavoro settimanali e delle otto ore giornaliere. Dall'altro vi era l'irruzione sulla scena sociale della soggettività operaia che, non solo alimentava movimenti sociali di contestazione globale, ma nel contempo riempiva di senso il tempo di non lavoro inventando pratiche di tempo libero e di turismo creative e non subalterne alla cultura capitalistica. Entrambi questi due processi sociali, coniugati insieme, facevano pensare ad una società in cui il lavoro perdeva progressivamente centralità. nel conferimento del senso di appartenenza sociale del soggetto, nella declinazione della sua cittadinanza, nella costruzione della sua identità sia soggettiva che sociale, mentre lo acquistavano fenomeni sino a quel momento considerati ai margini come il consumo e il tempo libero.

Nell'esprimere comprensione per questo abbaglio teorico, che derivava da motivi anche nobili e condivisibili, come il desiderio di vedere crollare un sitema politico e sociale che aveva prodotto alineazione e sfruttamento in una misura che in quel momento appariva del tutto insopportabile a grandi masse di lavoratori, vogliamo mettere in evidenza un tema che ritornerà spesso nella nostra esposizione. Tutta la teoria del "post-industriale", tenuta sotto controllo dai maestri, ma debordata rapidamente in esaltata retorica polemica nei suoi molti epigoni, ha fatto da specchio deformante che, sopravvalutando fenomeni di breve periodo, ha impedito di vedere le grandi trasformazioni che si stavano verificando in quegli stessi anni.

La deformazione della realtà deriva da una quetsione che è tutto sommato abbastanza semplice. La progressiva riduzione quantitativa della durata del tempo di lavoro, rispetto al tempo di non lavoro, può voler dire la progressiva perdita di importanza del lavoro come fattore interpretativo fondamentale dei rapporti sociali, ma non vuol dire questo necessariamente. In un sistema produttivo in cui la capacità di valorizzazione del lavoro operaio cresce esponenzialmente, per effetto della crescente automazione dei processi produttivi, la quantità di capacità di valorizzazione 
erogata in termini di tempo diventa progressivamente sempre meno rilevante. I lavoratori, sempre più professionalizzati, del settore centrale dell'economia moderna hanno un potere contrattuale cosi elevato da poter forzare la stessa durata della giornata lavorativa e puntare, per esempio in Francia, all'obiettivo della giornata di 35 ore. L'avere più tempo libero a disposizione però, non è detto che significhi necessariamente che questi lavoratori perdano la loro identità operaia per cominciare ad identificarsi con quella dei ceti medi che, tanto per fare un esempio, possono fare le vacanze fuori stagione. L'autonomia e la consapevolezza che questo processo di progressiva marginalizazzione del tempo di lavoro comporta si gioca su più tavoli e non necessariamente su quello della trasformazione dei lavoratori in ceto medio.

Ma torneremo più avanti su questo concetto.

Quello che qui ci preme sottolineare è un'altra questione che questa polemica sottende e deforma. Il "lavoro saariato" costituisce una delle categorie sociali che ha un carattere più spiccatamente fondativo della società moderna: la perdita di centralità del lavoro spinge i teorici del post-industriale ad aprire la strada alla teoria del post-moderno e a tutta quella lunga schiera di studi che hanno sottolineato la crisi della modernità nel trentennio che va dagli anni Ottanta ai primi anni del nuovo millennio.

La crisi dei linguaggi artistici ha fatto da apripista ad una riflessione scarsamente fondata sull'analisi empirica e che ha un carattere smaccatamente ideologico. La comparsa dell'arte e dell'architettura post-moderna ha incoraggiato anche $i$ più riluttanti a lasciarsi andare nella critica della modernità ed a trovare tracce funzionali a questa critica anche in processi che avevano ben altra motivazione e spiegazione.

Non avendo in questa sede il benché minimo intento polemico eviteremo di entrare nel merito dei molti luoghi comuni su cui si gioca questa mistificazione. Preferiamo d'altro canto soffermare la nostra attenzione su quegli autori che peccano solo per eccesso di 
desiderio di cambiamento, piuttosto che su quelli che costruiscono comodi pretesti retorici per i partiti politici "nuovisti" che si affacciano sulla scena politica europea con il crollo della "cortina di ferro" e la fine della guerra fredda in Europa 4.

La questione che nessuno di questi autori riesce a mettere in crisi è legata ad un'idea che, i più avveduti e colti (per apparire particolarmente sofisticati), denominano come "tipicamente tedesca": l'idea cioè che il lavoro, la concezione del lavoro, la cultura del lavoro sia in grado di caratterizzare l'intera struttura sociale in quanto attività fortemente dotata di senso. E' vero che questa idea è "profondamente tedesca" in quanto è presente non solo nel Marx del Capitale ma anche in quello dell' "Ideologia tedesca" e dei Grundrisse, ma anche in Hegel ed in moltisismi altri autori della scuola hegeliana. Ma dire che è un'idea tedesca non può voler dire che si tratta di un'idea ottocentesca, superata dall'evoluzione della storia dei secoli successivi.

Scegliamo qui di confutare una tale affermazione, piuttosto che su elementi speculativi, sulla semplice constatazione dei temi del dibattito corrente nel corso dell'ultimo trentennio. E' vero che nello scontro politico italiano il lavoro è sempre più marginale e gli operai non sono certamente il ceto sociale che fa da forza aggregante, ma è vero anche che di lavoro si parla sempre e comunque, nelle forme più diverse e articolate. E si parla non solo dell'assenza di lavoro ma soprattutto di tutte quelle forme di lavoro, flessibile, interinale, a progetto, di collaborazione, che consentono di evitare la creazione di rapporti di lavoro stabili e continuativi, regolari e garantiti. In un quadro, quindi, perdente e subalterno, la questione del lavoro ritorna continuamente a riproporsi come pietra di paragone e termine di confronto ineludibile.

Tanto più che la diminuzione della durata della giornata lavorativa al di sotto delle quaranta ore settimanali, loddove vi è stata, piuttosto che portare all'esplosione della creatività

4 Possiamo considerare un caso l'adesione di diversi architetti postmodernisti alla corte del craxismo vincente nel cuore degli anni Ottanta? 
vacanziera, ha portato all'esplosione del secondo e del terzo lavoro, alla creazione di attività di lavoro indipendente ed autonomo, alla costruzione di filiere professionali innovative che consentono elevati livelli di produttività al riparo del sistema pubblico delle garanzie. Non parliamo qui solo dei lavoratori in cassa integrazione, che certamente in questi fenomeni di "lavoro diffuso" possono riconoscersi, ma soprattutto dell'area del pubblico impiego ed in generale del lavoro garantito.

Dai rapporti sociali di produzione discende una determinata forma di società, e questo perché la concezione del lavoro che prevale in una organizzazione sociale specifica rappresenta un "fatto sociale totale" 5 , un modo di intendere le relazioni sociali, le forme di aggregazione, e persino la cultura, il diritto, l'arte, la letteratura e via dicendo. Negli anni in cui l'impianto della Fiat Mirafiori, a Torino, rappresentava la più grande fabbrica italiana, i rapporti sociali che erano presenti in quella fabbrica erano i medesimi di quelli esistenti nell'intera società torinese, piemontese e forse italiana. Non è un caso che in quegli stessi anni Torino fosse la città più avanzata d'Italia nel campo della comunicazione, dell'arte e della cultura; la città in cui ha preso le mosse l'industria cinematografica, la radio prima e la televisione poi, la città di Sironi e Felice Casorati.

\section{A partire dalla flessibilità}

Con gli anni Ottanta la critica all'alienazione del lavoro operaio comincia a prendere una forma nuova, fino a quel mometo sconosciuta. La prevalenza del lavoro stabile e garantito rende difficile dare risposte alla domanda di lavoro fluttuante che nasce nelle imprese a forte variazione stagionale del ciclo produttivo,

5 Parliamo qui di "fatto sociale totale" nell'accezione che M. Mauss ha dato a questo termine nella sua opera.

Cfr. M. Mauss, Manuale di etnografia, Jaca Book, Milano 1969; M. Mauss, Sociologia e antropologia, Newton Compton, Roma 1976; M. Mauss, I fondamenti di un'antropologia storica, Einaudi, Torino 1998; M. Mauss, Saggio sul dono, Einaudi, Torino 2002. 
oppure a forte varianza del mercato. Il problema nasce da un fondamento che è tutt'altro che pretestuoso. La mutevolezza dei gusti del consumatore affluente induce la nascita e lo sviluppo di segmenti di mercato a fortissima varianza della domanda. Non si possono produrre più prodotti altamente standardizzati6, ma si deve introdurre una varianza di modelli che consenta al consumatore l'illusione del prodotto personalizzato. Di più: si deve avviare un ciclo produttivo sapendo che l'attività routinaria non può che durare alcuni mesi e solo in alcuni limitatissimi casi pochi anni. La norma diventa il cilco produttivo ad alto livello di varianza, flessibile, pronto a rispondere ai cambiamenti di mercato.

Con questa motivazione, del tutto illogica e niente affatto necessaria, si sviluppa l'offensiva contro il lavoro stabile e garantito che era tipico della società industriale che, dal secondo dopoguerra agli anni Settanta, si era sviluppata nel nostro paese. A produzione flessibile deve fare riscontro un rapporto di lavoro flessibile, in cui licenziamento e mobilità siano modalità cui si possa fare ricorso agevolmente, senza troppe complicazioni. Nel caso italiano sul fronte del licenziamento si riesce a resistere per qualche decennio, anche grazie al meccanismo della cassa integrazione, mentre su quello della mobilità, sia orizzontale che verticale, si sviluppano strategie d'impresa che rendono il posto di lavoro, ed il contrattodi lavoro che lo regola, una vera e propria finzione giuridica.

Se non diamo per scontato il fatto che la produzione flessibile non si debba coniugare necessariamente con il lavoro flessibile, ci rendiamo facilmente conto di quanto vi sia di strumentale in questo dibattito. Il problema vero è che la grande fabbrica è diventata politicamente rigida, e gli alti livelli di sindacalizazzione e di capacità di mobilitaizone attiva rendono impossibili le nuove strategie di gestione della forza lavoro. Non ci sono solo problemi

6 "Compratela di qualsiasi colore purchè sia nera". H. Ford forse non ha mai pronunciato questa frase, che rende molto bene l'idea di standardizzazione del prodotto che aveva al suo interno il modello fordista. 
di costo del lavoro e di rivendicazioni contrattuali, ma c'è soprattutto la facilità con cui può essere bloccata l'attività di impianti giganteschi, fortemente centralizzati. La grande fabbrica ha smesso di essere fonte di economie di scala ed è diventata fonte di diseconomie.

La stagione del decentramento produttivo manda rapidamente in frantumi un mercato del lavoro che aveva strutturalmente un tasso di disoccupazione molto basso, in cui la stabilità del posto di lavoro era notevole, in cui la fedeltà all'impresa del lavoratore era pressocchè assoluta e si esprimeva nell'accettazione di un modello sostanzialmente familistico e paternalista7. E' in questi anni che il mercato del lavoro italiano 8 muta rapidamente la propria caratteristica struttura, e progressivamente diventa un mercato caratterizzato da:

- notevole disoccupazione occulta (grazie alla Cassa integrazione);

- pressocchè nullo tasso di reimpiego dei lavoratori espulsi;

- scarsisima propensione alla creazione di nuovi posti di lavoro.

Mentre la flessibilità era stata invocata per potere meglio creare nuovi posti di lavoro, nei cicli produttivi qualificati che le nuove produzioni consentivano, nei fatti si traduce nella distruzione di posti di lavoro stabili e regolari, per la loro sostituzione con posti di lavoro temporanei o precari, quando non a condizioni irregolari. L'abbandono del modello garantista doveva servire a creare fluidità fra i diversi segmenti del mercato del lavoro9,

7 Da un punto di vista sindacale, che oggi possiamo ben giudicare miope, sono queste le critiche che vengono rivolte al modello olivettiano che in questi anni si sviluppa e contagia la cultura d'impresa di esponenti dell'imprenditorialità italiana di primo piano come i Pirelli o i Crespi.

8 Cfr. E. Reyneri, Sociologia del mercato del lavoro, Il Mulino, Bologna 2002; E. Pugliese, E. Rebbeggiani, Occupazione e disoccupazione in Italia: dal dopoguerra ai giorni nostri, Il Mulino, Nologna 2004.

9 Nei primi anni Ottanta c'era chi parlava addirittura di "balcanizzazione" del mercato del lavoro, usando un termine che di lì a poco sarebbe divenuto sinonimo di lotta cruenta e fratricida. 
troppo incrostati da degenerazioni burocratiche. Viene usato invece per produrre precarietà e ricatto, insicurezza e deprofessionalizzazione. I posti di lavoro stabili e regolari della grande fabbrica vengono gradualmente sostituiti con posti di lavoro precari, spesso irregolari, mal pagati, fondati sul ricatto della facile licenziabilità. Si inizia distruggendo il rispetto dell'orario di lavoro e non pagando gli straordinari e, a poco a poco, salta ogni garanzia e le pretese si accrescono fino a toccare forme di vero e proprio arbitrio. Il part-time imposto alla donne in maternità quando non addirittura la lettera di dimisisoni firmata in bianco, forme di cottimo più o meno occulte, lavoro festivo o rischioso non pagato, la formazione obbligatoria a proprie spese, il mobbing e così via.

Il campionario dell'arbitrio padronale che abbiamo potuto costruire in questi anni con le nostre ricerche è talmente ampio che è molto difficile giustificare come sia stata possibile una così spregiudicata distruzione del sistema pubblico delle garanzie del lavoro. Ci è capitato persino, in un'occasione di confronto internazionale, di dovere subire le critiche incredule di colleghi troppo amanti dell'Italia da potere accettare un così primitivo stato di cose. Che all'origine di ciò vi sia sicuramente una cultura imprenditoriale che stenta ad uscire dalla sua fase avventurosamente pionieristica è certamente vero, anche se ad essa fa sicuramente riscontro una cultura sindacale che ha un livello di disponibilità al compromisso che è del tutto ingiustificata.

La spiegazione che ci siamo dati è che concorrono due fenomeni che sono molto complessi entrambi. Il primo è la crisi della giustizia, con il progressivo allungarsi dei tempi processuali, anche per ciò che attiene ai procedimenti lavoristici. Il controllo sindacale e le lotte operaie degli anni Settanta avevano imposto un progressivo accorciamento dei tempi dei procedimenti, cui aveva concorso anche una giurisprudenza che era generalmente favorevole alle ragioni operaie e sindacali. Cogli anni Ottanta, il mutato clima politico ha comportato un graduale smottamento della giurisprudenza a favore del punto di vista delle imprese, un 
progressivo allungarsi dei tempi dibattimentali e, quel che è peggio, il prevalere della logica arbitrale e transattiva. Oggi le controversie di lavoro si risolvono molto spesso con una transazione che non rientegra il lavoratore, anche quando ha subito le peggiori ingiustizie, ed in cui i lunghi tempi processuali servono a fargli accettare una transazione pecuniaria ben al di sotto di quella prevista dal sistema pubblico delle garanzie. Anche senza chiamare in causa la crescita, soprattutto negli ambienti professionali, della corruzione degli ultimi anni, ci si può rendere facilmente conto di quale risultato degenerativo ha indotto l'introduzione dell'istituto del patteggiamento nelle aule giudiziarie.

\section{La precarizzazione del lavoro}

Ma gli esiti sempre negativi dei processi del lavoro, pur avendo avuto un ruolo notevole nel produrre obbedienza anche in un quadro di irregolarità delle condizioni di lavoro, non poteva indurre gli effetti devastanti di precarizzazione dei rapporti di lavoro che sono oggi sotto i nostri occhi. L'altro versante del problema è, infatti, rappresentato dalla forte diminuzione della domanda di lavoro de-professionalizzato che l'innovazione tecnologica ha indotto nel ciclo produttivo.

L'informatizzazione della fabbrica e la comparsa delle macchine a controllo numerico ha comportato una ristrutturazione produttiva che ha ridotto notevolmente il fabbisogno di lavoro manuale tradizionale, introducendo una nuova domanda di attività di controllo e manutenzione, che nel contempo distruggono un gran quantità di posti di lavoro tradizionali e ne creano in sostituzione un numero di gran lunga più piccolo. Da qui la difficoltà di reimpiegare chi viene licenziato, e che non possiede spesso le caratteristiche tecniche e professionali adatte alla riconversione. Comprensibile che, in questo quadro rientri la sostituzione di forza lavoro avanti nell'età, sindacalizzata e per queste due ragioni costosa, con forza lavoro giovane, difficilmente sindacalizzabile, e per queste due ragioni meno costosa. 
Incidentale che, in questo quadro, i lavoratori giovani sono in molti casi anche lavoratori stranieri, di recente immigrati, in condizioni di anomia sociale e quindi fortemente ricattabili

Due ragioni quindi, per un processo di precarizzazione del lavoro industriale che è unico. Da un lato la difficoltà di fare ricorso al sistema giudiziario, dall'altro la ridotta domanda di lavoro professionale, costruiscono una situazione in cui i lavoratori del settore garantito hanno difficoltà a difendere il sistema delle garanzie, e finiscono per rendersi partecipi dello smantellamento complessivo del sistema a causa della loro debole capacità contrattuale.

Per comprendere meglio il quadro che si è venuto a creare, e che ha disarmato ogni velleità di resistenza, è sufficiente aggiungere che le condizioni complessivamente ricattatorie che abbiamo sin qui descritto vanno coniugate con il quadro, sostanzialmente corruttivo che il sindacato italiano ha dovuto suo malgrado favorire, accettando la dilatazione dell'uso della cassa integraizone, ben al di là delle sue motivazioni istituzionali. Garantendo le retribuzioni dei nuclei operai contrattualmente più forti, a spese del contribuente pubblico, si è finito per avallare un sistema in cui il consenso operaio viene comprato attraverso un trattamento assistenziale dilatato per via amministrativa e che non rispetta più gli orginari ordinamenti. Nel 2011 la "Cassa Integrazione Guadagni" si avvia a raggiungere i tre miliardi di ore erogate, un record assoluto che consente anche ai lavoratori di partecipare alla spartizione della grande torta del debito pubblico.

Come era accaduto nel trentennio 1945-75, quando i rapporti sociali di produzione nati e sviluppatisi nella fabbrica si erano diffusi a tutta la società, così anche nel trentennio successivo il tramonto del sistema delle garanzie del lavoro operaio si diffonde e si genereralizza, estendendo a tutta la società un processo di precarizzazione dei rapporti sociali che è ormai senza confini e si estende ben al di là del lavoro industriale propriamente detto.

Ovunque, non solo nella fabbrica, ma nelle PMI, nelle aziende artigiane, nel commercio e nei servizi, e persino nel pubblico 
impiego, si diffonde la pratica di distruggere posti di lavoro stabili e regolari, per sostituirli con posti di lavoro precari, a tempo parziale, a termine, quando non addirittura a condizioni irregolari. Immensa è la casistica di imprese che licenziano senza che nessuno sia in grado di imporre il divieto, che pure esiste nella nostra legislazione, di riassumere a condizioni di finto subappalto o di precarietà. Infinito l'elenco delle imprese, anche pubbliche, che usano illegittimamente stage e tirocini, convenzioni e gare d'appalto, per abbassare ingiustificatamente il costo del lavoro licenziando o mettendo in pre-pensionamento lavoratori regolari.

La distruzione del sistema pubblico di garanzia del lavoro dipendente porta con se non soltanto la fine del lavoro salariato regolare, ma la crsi della cultura del lavoro che quel modello portava implicitamente a compimento. La società moderna è una società che individualizza10 i rapporti sociali, che distrugge le formazioni sociali tradizionali, e differenziando i ruoli sociali, individualizza le relazioni in un processo che all'estremo contiene il germe di quell'anomia sociale che già Durkheim 11 indicava come causa della crescita del comportamento suicidario tipico delle società moderne.

Quello cui abbiamo assistito in questi anni è una spinta in avanti del processo di indidualizzazione dei rapporti sociali. La pretesa delle imprese di subordinare ai propri interessi la vita dei lavortori, di spingere i giovani ad "andare dove il lavoro chiama", ha comportato una più accentuata crisi di alcune istituzioni sociali fondamentali, si pensi alla famiglia, ma anche alla cittadinanza locale, con un processo di precarizzazione dei rapporti sociali che produce immediatamente crisi della natalità e della fertilità, abbandono delle generazioni anziane, spopolamento o addirittura desertificazione delle aree territoriali più marginali, crisi identitarie e molto altro ancora.

10 Cfr. N. Elias, La società degli individui, Il Mulino, Bologna 1995.

11 Cfr. E. Durkheim, Il suicidio. Studio di sociologia, BUR, Milano 2002. 
In generale la precarizzazione dei rapporti sociali comporta una spinta in avanti del processo di differenziazione sociale, senza produrre nel contempo quella carica solidaristica di cui la società avrebbe bisogno per "tenere insieme i pezzi". Anzi, al contrario, puntando tutto sulla competizione e sull'arrivismo individualistico, fa venir meno quel retroterra di solidarietà empatica che i legami familiari e amicali generalmente comportano. Per trovare lavoro si abbandona la famiglia d'origine, si posterga la creazione di una nuova famiglia, si attenuano i contatti con gli amici, i compagni di scuola, i vicini di casa, si rinuncia alla riproduzione sociale creando cosi non soltanto "quell'uomo a una dimensione" che già preoccupava Marcuse12, ma quel lavoratore senza cittadinanza che costituisce il presupposto sociale necessario per la realizzazione del totalitarismo moderno.

\section{Crisi economica e crisi sociale}

Abbiamo richiamato il saggio di Marcuse, che tanta fortuna ha avuto negli anni della nostra formazione, provocando la mobilitazione di grandi masse di giovani che in quella fase storica si affacciavano, spesso per la prima volta, sulla scena della formazione universitaria, perché rappresenta una buona pietra di paragone della difficoltà con cui ci si è resi conto di ciò che stava accadendo in quegli anni nelle società moderne. L'uomo a una dimensione è l'uomo consumatore, dimentico del proprio status di cittadino lavoratore, che si lascia strapazzare da un mercato che gli impone le sue scelte.

Nello sviluppo del mercato di massa degli anni Settanta, nella grande crescita dei mezzi di comunicazione e delle tecniche pubblicitarie, vi sono certamente aspetti che descrivono bene come si presentva la scena di quegli anni e quanti elementi di preoccupazione si addensavano nella mente di chi si interrogava

12 Cfr. H. Marcuse, L'uomo a una dimensione. L'ideologia di una società industriale avanzata, trad. di L. Gallino, Einaudi, Torino 1977. 
con consapevolezza sul futuro delle società industriali di massa. Per gli ebrei tedeschi, sfuggiti al nazismo e che approdando negli Stati Uniti, la potenza produttiva e l'intraprendenza comunicativa della giovane società americana, non poteva che allarmare per i suoi tratti implicitamente totalitari che erano già visibilissimi negli anni del secondo dopoguerra. Ma l'Europa pensava di essere al riparo, e nella critica militante europea al breve ma incisivo saggio di Marcuse c'era tutta l'arroganza del "vecchio continente", che si ritiene ormai vaccinato dalla guerra antinazista contro ogni forma di totalitarismo.

Abbiamo qui fatto più volte riferimento alla periodizzazione di M. Tronti 13 , che assegna un ruolo di cesura epocale al 1975, direi al quadriennio 1971/75. Senza entrare nel merito del carattere identificativo di questa periodizzazione ${ }^{14}$, possiamo dire subito che ci convince perché consnete di connotare con precisione il periodo dal 1975 ai giorni nostri15. Gli anni a venire ci diranno se stiamo vivendo un periodo omogeneo che da quella cesura deriva, oppure se non sia possibile identificare due periodi distinti; un ventennio 1975/1997 che rappresenta il ventennio in cui si carica la molla, in cui si innescano le tensioni finanziarie che esploderanno nel periodo dal 1997 ai nostri giorni, in una sequenza di esplosioni che non è ancora finita e di cui non si

13 Ci rifreiamo qui alla conferenza, tenuta da M. Tronti, il 17 novembre 2011, presso il Dipartimento di Scienze Sociali dell'Uniersità di Roma "Sapienza", nell'ambito del seminario organizzato dal gruppo GRADEC, in preparazione della III Conferenza Mondiale per la Decrescita di Venezia 2012.

14 Tralasciando quindi la disomogeneità che questa periodizzazione introduce rispetto a quella del "Secolo breve" di E. Hobsbawm.

15 Dobbiamo ancora aspettare qualche anno per capire se il trentennio finisce nel triennio 2008/2010, in cui la crisi finanziaria internazionale rappresenta sicuramente il tratto saliente, ma di cui tutti gli elementi erano presenti nelle precedenti crisi, per esempio russa, brasiliana o argentina (dicembre 2001), se non addirittura nella crisi dei mercati asiatici del 1997, che coinvolse paesi come la Thailandia, l'Indonesia, la Corea del Sud e lo stesso Giappone. 
intravede la fine. Per poterlo capire avremmo bisogno di intepretare con precisione il presente che stiamo vivendo e che è ancora molto contraddittorio e nebuoloso.

Ma in sostanza, cosa comincia nel quadriennio $1971 / 75$, con la denuncia degli accordi di Bretton Woods, la fine della convertibilità aurea del dollaro e i due "shock petroliferi"?

Innanzi tutto bisogna dire che finisce il clima liberistico e cooperativo che aveva carattizzato la fine della Seconda Guerra Mondiale. Per la conversione della propria economia di guerra gli Stati Uniti avevano adottato una strategia di integrazione euroamericana, di decolonizzazione, di intervento solidaristico in chiave anti-sovietica. La costruzione dell'impero, necessario per combattere la "guerra fredda", avviene sulla base di un'ideologia sostanzialmente solidaristica e keynesiana, che dal "new deal" roosveltiano coinvolgerà, col Piano Marshall, tutta Europa. Questa fase si conclude traumaticamente nel 1971/75, e si esplicita con forza con la comparsa sulla scena politica europea di Margaret Thatcher, divenuta premier britannico nel 1979, e di Ronald Reagan, che nel 1981 conquista la presidenza degli Stati Uniti.

Dal 1975 al 1981 va in gestazione quella ideologia liberista e antistatalista, che si ammanta di spirito riformista, ma che progetta e realizza riforme che smantellano pezzi importanti dello stato sociale e rilanciano l'accumulazione capitalistica attraverso la privatizzazione del patrimonio dello Stato, costruito negli anni dell'eroica "ricostruzione" del dopoguerra. In sostanza la teoria che sta alla base dell'idoelogia neo-liberista è che per vincere la non dichiarata "terza guerra mondiale", bisogna fare delle sostanziali riforme che stimolino le capacità di competizione dell'Occidente, che creino un clima interno di scontro securitario e terroristico 16 , che eliminino le garanzie consensualistiche del

16 Cfr. Huntington S., Lo scontro delle civiltà e il nuovo ordine mondiale, Garzanti, Milano 2000; Fukuyama F., La grande distruzione. La natura umana e la ricostruzione di un nuovo ordine mondiale, Baldini \& Castoldi, Milano 2001. 
"secolo socialdemocratico" caratteristiche della "vecchia Europa", per puntare alla creazione di un quadro di competizione globale che faccia crollare su se stesso il nemico bolscevico.

Senza entrare nel merito delle complesse situazioni storiche che stanno dietro a questo progetto politico, occorre rilevare qui quanto sia stato sottovalutato in Europa il senso ideologico e politico di questo complesso programma. Per affrontare e realizzare i compiti che ci si poneva, si architetta un vero e proprio progetto culturale, che ha nella competitività individualistica, nel privilegio fondato sul successo e nell'ideologia compassionevole i suoi fondamenti. Questa ideologia interpreta la sicurezza sociale come strumento di intervento per sostenere i bisognosi, quelli che restano indietro perchè non hanno la capacità di stare al passo con l'insieme della società, che non devono essere aiutati in quanto titolari di diritti, ma piuttosto in virtù della capacità di compassione di quei "forti e puri" che sono il nucelo centrale della società, quelli che sanno stare al passo con la competizione e che sono per questo i portatori del nuovo. Da qui lo smantellamento dello stato sociale per la costruzione di un "welfare minimo" che è fondato su di un sistema delle garanzie a carattere residuale.

La cosa che è importante ricordare è che questa ideologia non si presenta come conservatrice o reazionaria, ma si presenta come riformatrice, e deliberatamente intenzionata a "rivoluzionare" il vecchio ordine mondiale. Ciò a cui assistiamo in questa fase storica, a cominciare dalle crisi finanziarie del periodo $1997 / 2011$, che non a caso si aprono proprio in Asia, dove scalpitano le "giovani tigri" che minacciano il primato imperiale americano, non è che il precipitato di un processo di trasformazione sociale che si sforza di definire un nuovo equilibrio imperiale. Il ridimensionamento del ruolo del Giappone e delle economie delle "giovani tigri" asiatiche, non è stato che il primo passo di una complessa strategia che ora punta a ridimensionare il ruolo dell'Europa e della sua fortissima moneta. Questa operazione politico-economica non può che essere al tempo stesso anche culturale e sociale. Deve impedire che vada 
avanti il processo di unificazione europea, deve contrastare tutti quegli elementi ideologici e politici, su cui era fondata la supremazia del "vecchio continente": solidarismo, democrazia consensuale, politiche economiche redistributive, pluralismo politico, alternanza democratica.

In definitiva la crisi finanziaria, come crisi economica e sociale al tempo stesso, come movimento di trasformazione dei rapporti di forza per riprendere un'egemonia che si rischia di perdere. Troviamo in questo la motivazione di quell'attacco alla cultura del lavoro che è tipicamente europea. Che cos'è se non questo l'emergere di un mercato finanziario che realizza grandi fortune con un semplice spostamento di capitali, che ridicolizza gli sfrozi del buon padre di famiglia di insegnare la cultura del lavoro ai propri figli, mostrando esplicitamente le incredibili ricchezze che si possono realizzare giocando sui derivati, o creando prodotti finanziari truffaldini, che però si evita accuratamente di regolamentare, come i fondi sub-prime.

La sfida finanziaria ha in radice una sfida culturale che vuole distruggere la cultura del lavoro come primo elemento ideologico su cui costruire una società fondata sulla competizione globale e sul trionfo della forza e della spregiudicatezza individuale. Le sfide che l'Occidente ha di fronte sono mortali e per vincerle deve attrezzarsi con strumenti di guerra. La cultura cristiana della pace e del dialogo interculturale sono i principali nemici interni, che bisogna contrastare a tutti i costi, per potere attrezzarsi adeguatamente per le sfide future cui occorre far fronte.

Quello che abbiamo avuto difficoltà a capire per tempo è che avevamo di fronte un nemico ben più lucido e determinato di quanto non fosse percepibile in quella fase storica. Noi pensavamo di esser di fronte alla strategia politica di un leader improvvisato, come l'ex-attore Ronald Reagan, ed invece eravamo davanti alla costruzione politica di un gruppo dirigente vero e proprio, dotato di forti radici sociali e capace di una grande 
progettualità strategica. Il vecchio Ike Eisenhower 17 , in tempi non sospetti, aveva messo in guardia il mondo intero del pericolo che, quello che lui chiamava "complesso militare-industriale" rappresentava, e rappresenta ancora oggi, per la democrazia. Le guerre balcaniche $(1991 / 95,1996 / 99)$ e le due guerre del golfo (1990/91, 2003), ci hanno dimostrato negli anni successivi quanto fosse forte e spietata la determinazione di questo gruppo dirigente che minaccia ogni giorno di più, e con tutte le armi a sua disposizione, la pace nel mondo.

La varietà dell'arsenale di armi impiegato e la cosa che colpisce di più l'ideologia pacifista della "vecchia europa". Prima l'arma della competizione militare/culturale con l'est sovietico, poi quella del conflitto ideologico/religioso con il nemico medio-orientale che controlla le fonti di approvvigionamento petrolifero, oggi infine quella della competizione finanziaria. Siamo insomma in un contesto di crisi economica, politica, sociale globale, in cui non c'è distinzione alcuna fra nemico interno e nemico esterno; chi la conduce non fa prigionieri e non accetta limiti alla sua volontà di potenza.

E' ancora troppo presto per dire se le crisi finanziarie del periodo 1997/2011 fanno parte integrante della fase storica che si inizia con il reganismo, o se non siamo di fronte ad una fase nuova, che propone un deciso scatto in avanti della sfida per l'egemonia mondiale. Gli anni a venire ce lo diranno e speriamo per quella data di essere ancora in condizioni di poterlo raccontare.

17 Generale dell'esercito statunitense vincitore della Seconda Guerra mondiale e $34^{\circ}$ Presidente degli Stati Uniti ha più volte, nei ultimi anni della sua vita politica, sottolineato quanto fosse pericoloso, per la democrazia, lo strapotere di un "complesso militare-industriale" che ha continuamente bisogno di guerre per alimentare se stesso e la propria forza di controllo e di influenza sul mondo. Cfr. Farewell Address del 17 gennaio 1961. 


\section{La finanziarizzazione dell'economia mondiale}

Nel corso degli ultimi anni da più parti si sottolinea il carattere del tutto nuovo della crsi economica che l'Occidente sta attraversando, ricordando in primo luogo il fatto che i livelli raggiunti dalla finanziarizzazione dell'economia mondiale sono assolutamente inusuali.

La classica equazione marxiana $M-D-M$, nel trasformarsi nell'equazione D - M - D', mostra con chiarezza come il processo di valorizzazione del capitale non usa solo il denaro come semplice segno del valore della merce ma come strumento vero e proprio di accumulazione. La qustione che è però centrale nella situazione attuale è che ci troviamo di fronte alla produzione di danaro a mezzo di denaro. In fondo questo intendiamo per finanziarizzazione: una circolazione di moneta vera e propria o virtuale, che genera valore attraverso lo scambio di Borsa, con una sua specifica dinamica domanda/offerta, che non ha relazione alcuna con il sistema della produzione di beni e servizi.

Marx sapeva bene che già Cosimo I de Medici aveva fondato la sua potenza ed il suo potere proprio sulla produzione di denaro a mezzo di denaro. Proprio per questo, nel capitolo del Capitale che affronta il temna dell'accumulazione originaria, sottolinea i passi avanti compiuti dall'Italia signorile del XIV secolo sul cammino della transizione dal feudalesimo al capitalismo 18 .

La questione però viene bene in luce con Pierre Vilar, nelle sue fondamentali ricerche sullo sfruttamento delle risorse di minerali preziosi in America Latina da parte dell'impero asburgico di Carlo $\mathrm{V}$ e dei suoi successori19. L'elemento che Vilar non si stanca mai di sottolineare è che la moneta non è che una merce, prova ne sia che il suo valore oscilla, come quello di ogni altra merce, al

18 Cfr. K. Marx, Capitolo 23, L'accumulazione originaria, Primo libro, Il capitale.

19 Questi studi erano ben presenti a F. Braudel, alla pubblicazione del suo Civiltà e imperi nel Mediterraneo all'età di Filippo II, Einaudi, Torino 1986. 
variare del rappprto fra domanda ed offerta 20 . La grande spinta inflattiva che porterà al crollo dell'impero asburgico ha la sua origine nell'uso scriteriato dello strumento del debito e farà la fortuna delle grandi famiglie bancarie sia italiane che fiamminghe, fra l'altro indirettamente finanziando la rivoluzione delle Provincie Unite ed il loro sviluppo in senso capitalistico degli anni successivi.

Ricordiamo tutto questo perché oggi si torna a parlare di una sorta di dicotomia capitalistica che ha poco senso. Da un lato vi sarebbe il capitalismo buono, produttivo, fondato sul commercio di beni e sulla loro produzione, dall'altro vi sarebbe il capitalismo cattivo, parassitario, prodotto dalla finanziarizzazione dell'economia e dal prevalere del potere bancario. Sterminato è il numero degli esempi che si possono fare per dimostrare l'intreccio strettissimo che vi è fra capitalismo cosiddetto produttivo e capitalismo cosiddetto parassitario. Vi è persino chi si spinge a teorizzare l'esistenza di una sorta di ricorsività nel ciclo dell'accumulazione, che passa di settore produttivo in settore produttivo e, quando consuma tutti i suoi spazi di manovra, da ultimo abbandonando il settore immobiliare, si rivolge alla speculazione finanziaria. Quella in sostanza che stiamo vivendo sarebbe insomma la "madre di tutte le crisi", quella crisi assoluta che conclude il circuito della storia.

Lo scetticismo con cui racconto questa tesi è dovuto alla semplice constatazione di quanta prudenza sia necessaria per entrare in una logica previsionale, sia essa di breve, medio o lungo periodo. La storia ci ha sorpreso tante di quelle volte che ormai la prudenza è d'obbligo. Tanto più che le dimensioni quantitative del fenomeno sono così grandi da consigliare un atteggiamento di paziente attesa.

Oggi la massa monetaria circolante, sia essa propria o impropria, ha raggiunto dimensioni inusitate e ad essa si somma una massa finanziaria virtuale che supera di gran lunga la dimensione

20 Cfr. P. Vilar, Oro e moneta nella storia: 1450-1920, Laterza, Bari 1971. 
complessiva del PIL globale. Insomma è andato in crisi il rapporto fra finanza ed economia reale, e la produzione di moneta vurtuale è diventata fine a se stessa, e si autoriproduce senza tenere in alcun conto i processi di produzione di quella che una volta si chiamava la "ricchezza sociale". L'incredibile fantasia con cui si producono strumenti finanziari cosiddetti "derivati", dei quali è molto difficile controllare il valore intrinseco, e che vengono quotati ed offerti ai risparmiatori senza essere in grado di dare un valore che non sia quello figurativo delle borse, dimostrano come siamo di fronte ad un mercato che è del tutto auto-referenziale, che non ha nulla a che vedere con l'economia reale. Anche se poi, con i suoi movimenti inconsulti, è perfettamente in grado di mandarlo in crisi in ogni momento, con la stessa dimensione ordinaria dlele proprie fluttuazioni.

Ciò non vuol dire naturalmente che si tratta di un mercato separato, che non ha implicazioni sull'insieme del mercato finanziario, ed anche sul mercato delle merci vere e proprie. I mercati sono intercomunicanti, e non vi sono barriere in grado di separarli e di renderli ermeticamente isolati dal resto del sistema finanziario. Un prodotto, una volta che si diffonde in una piazza finanziaria specifica è, per ciò stesso, presente in tutte le altre e risente delle loro dinamiche simultaneamente. Da qui l'incredibile groviglio di processi di causazione circolare di fornte a cui si trovano gli stati più indebitati del pianeta. Nel momento in cui emettono titoli per rifinanziare il proprio debito debbono necessariamente accedere al mercato dei capitali e, con ciò stesso diventano partecipi di quelle dinamiche che sono in atto, per effetto delle scelte di attori finanziari di mercato, politicamente ed eticamente irresponsabili, anzi che fanno della loro irresponsabilità una bandiera di libertà.

In conclusione la nostra interpretazione della crisi finanziaria che stiamo vivendo è estremamente ottimistica e pessimistica al tempo stesso. Da un lato non pensiamo di essere di fronte ad un feneomeno del tutto nuovo, se non per le dimensioni quantitative che ha assunto. Il denaro virtuale non è nient'altro che una merce nuova, che arriva sul mercato in dimensioni massiccie per la 
prima volta, ed il cui commercio, come è avvenuto per tutte le altre merci, va attentamente regolamentato. E' questa assenza di regolamentazione che ne ha reso esponenziale la produzione, senza che sia possibile verificare la qualità intrinseca del prodotto e, da che mondo e mondo la "moneta cattiva ha scacciato sembra quella buona". Ciò che ha reso possibili ogni tipo di truffe 21 è proprio questa assenza di regolamentazione.

E questo è il lato ottimistico della faccenda. Si tratta di una semplice assenza di regolamentaizone che le autorità politiche possono sforzarsi di affrontare dotandosi dei più adeguati strumenti di controllo. E' difficile, è molto complesso, ma possiamo provare a dotarci di quegli strumenti che sono necessari per provarci, tanto più che a volte basta annunciare un provvedimento per vederlo applicato ancora prima che sia necessario provvedere alla repressione, e questo è esattamente il caso degli strumenti finanziari e bancari.

La questione vera, che ci fa diventare pessimisti è un'altra. Per ottenere un adeguato risultato non bastano strumenti di controllo a carattere nazionale, ma occorrono strumenti di controllo a carattere sovra-nazionale, ad una scala di potere rispetto alla quale non esistono in questa fase storica adeguati livelli di autorità. La finanza è integrata su scala mondiale, il potere è disarticolato su scala nazionale e gli organi di governo sovranazionali hanno un deficit di autorità che è sotto gli occhi di tutti. Banca Mondiale, FMI, BCE provano costantemente a dire la loro, ma il difetto di legittimazione limita talmente la loro capacità di agire da riuscire a produrre risultati molto scarsi22. Il deficit di autorità è così grande che si trovano a combattere contro un

21 Pensiamo qui alle cosiddette "piramidi finanziarie" albanesi, così come alla bolla immobiliare statunitense e al mercato dei fondi sub-prime.

22 Il cosiddetto "commissariamento" della Grecia e dell'Italia, che ha portato alle dimisisoni dei governi usciti vincitori dai confronti elettorali, e alla nascita di governi di unità nazionale, mostra come le pressioni delle autorità sovranazionali non sono certamente ininfluenti, anche se i risultati concreti di queste pressioni sono del tutto discutibili ed ancora tutti da costruire. 
nemico potentissimo, le grandi concentrazioni finanziarie, con le mani legate dietro la schiena.

Colmare il gap esistente fra fabbisogno pubblico di governo dell'economia mondiale ed anarchia dei mercati, nonostante sia urgentissimo, è tuttavia molto difficile. Le forze sociali e politiche sono fortemente condizionate da questioni di retroguardia ed hanno una scarsa capacità di interpretare il momento politico che stiamo vivendo. E' questo che ci rende profondamente scettici e pessimisti sulle urgenze che sono davanti ai nostri occhi in questi mesi. Avremmo bisogno di istituzioni di livello sovra-naizonale, se non addirittura mondiale, per fare fronte all'emergenza, ed invece ci arrabattiamo ancora con le più sterili polemiche a carattere nazionale, con il particolarismo localistico, con un regionalismo di retroguardia che ci rende completamente esposti ai colpi della crisi che abbiamo di fronte.

\section{In conclusione}

La parziale e del tutto provvisoria conclusione a cui il nostro ragionamento conduce è a questo punto abbastanza evidente. Per rovesciare il tavolo della discussione che in questo momento sta avvenendo in tutte le economie più avanzate del pianeta occorre ripartire dal lavoro, dalla sua capacità di conferire senso all'esistena umana, dai diritti di cittadinanza che esso alimenta nella società.

Nel corso degli ultimi due secoli l'interlocutore politico dei diritti di cittadinanza dei lavoratori è stato lo stato-nazione, nella forma storica che si è consolidata nel corso delle rivoluzioni liberali dell'Ottocento. Oggi è questo il rapporto che è entrato in crisi irreversibile e che non è in grado più di assicurare risultati che siano adeguati alle emergenze che abbiamo di fronte. Sono nati organismi sovra-nazionali che non sono certamente all'altezza dei loro compiti, e le forze sociali stentano ad abbracciare con convinzione il percorso del loro rafforzamento politico. Così come non nasce un movimento europeo dei lavoratori, allo stesso modo stenta a nascere un'Europa politica capace di porre un freno alla 
strapotere della speculazione finanziaria. I mercati finanziari si sono integrati su scala mondiale e a questo livello di scala territoriale pongono le loro sfide, mentre i lavoratori balbettano le loro richieste su scala nazionale, quando addirittura non sono costretti a regredire su scala locale, a causa della timidezza dei loro vertici nazionali.

Questa è la questione che si pone qui e ora. Se vogliamo uscire dalla crisi economica che il paese attraverso dobbiamo, per forza di cose porre il problema in chiave sovranazionale ed europeista. Occorre che il movimento dei lavoratori dica la propria su scala europea, assumendo con convinzione la scelta del rafforzamento politico dell'Unione, e ponendo con forza e determinazione la questione dello spazio europeo dei diritti sociali. Solo un movimento dei lavoratori che opera in chiave europeista può sperare di uscire dalla crisi spingendo in avanti la ruota della storia, che poi vuol dire semplicemente rafforzare anzicchè indebolire i diritti di cittadinanza dei lavoratori.

Enzo Nocifora

Vicedirettore del Dipartimento di Scienze Sociali Università di Roma "Sapienza" Via Salaria 113, 00198 Roma Tel. +39685768336 vincenzo.nocifora@uniroma1.it 


\section{Riferimenti bibliografici}

Balibar E., Noi cittadini d'Europa? le frontiere, lo stato, il popolo, Manifestolibri, Roma 2004

Balibar E., Wallerstein I., Razza, nazione, classe : le identità ambigue, Edizioni associate, Roma 1991

Barbalet J. M., Cittadinanza : diritti, conflitto e diseguaglianza sociale, Liviana, Torino 2000

Barcellona P., L'egoismo maturo e la follia del capitale, Bollati Boringhieri, Torino 1988.

Bauman Z., Dentro la globalizzazione : le conseguenze sulle persone, Laterza, Roma 2001

Bell D., Appunti sulla società post-industriale, Torino 1967;

Braudel F., Civiltà e imperi nel Mediterraneo all'età di Filippo II, Einaudi, Torino 1986.

Braudel F., La dinamica del capitalismo, Il Mulino, Bologna 1986.

Cavalli A., Le origini del capitalismo, Einaudi, Torino 1975.

Darhendorf R., Libertà attiva : sei lezioni su un mondo instabile, Laterza, Bari 2005.

Darhendorf R., La liberta che cambia, Laterza, Bari 1981.

De Masi D. (a cura di), L'avvento post-industriale, F. Angeli, Milano 1985.

Durkheim E., Il suicidio. Studio di sociologia, BUR, Milano 2002.

Elias N., La società degli individui, Il Mulino, Bologna 1995.

Emmanuel A., Lo scambio ineguale: gli antagonismi nei rapporti economici internazionali, Einaudi, Torino 1972.

Fearherstone M., Cultura del consumo e postmodernismo, Seam, Roma, 1994.

Fukuyama F., La grande distruzione. La natura umana e la ricostruzione di un nuovo ordine mondiale, Baldini \& Castoldi, 
Milano 2001.

Gallino L., Dizionario di Sociologia, Torino 1978.

Gallino L., Globalizzazione e disuguaglianze, Laterza, Roma 2000.

Germani G., Urbanizzazione e modernizzazione, Marsilio, Padova 1970.

Giddens A., Le conseguenze della modernità. Fiducia e rischio, sicurezza e pericolo, Il Mulino, Bologna, 1974.

Giddens A., Sociologia. Una introduzione critica, Il Mulino, Bologna 1983.

Giddens A., La costituzione della società, Ed. Comunità, Milano 1990.

Harvey D., La crisi della modernità, Il Saggiatore, Milano 1993.

Hobsbawm E. J., Lavoro, cultura e mentalità nella società industriale, Laterza, Roma-Bari 1986.

Hobsbawm E. J., Il secolo breve, Rizzoli, Milano 1998

Hollingswoth T. R., Lindberg L. N., La regolazione dell'economia americana: il ruolo di mercato, clan, gerarchia e associazione, sta in Magatti A., Azione economica come azione sociale, Il Mulino, Bologna 1995.

Huntington S. ,Lo scontro delle civiltà e il nuovo ordine mondiale, Garzanti, Milano 2000.

Kennedy P., Il mondo in una nuova era, Garzanti, Milano 2001.

Latouche S., Saggio sull'americanizzazione del mondo, Elèuthera, Milano 2002.

Lyorard F., La condizione post-moderna, Feltrinelli, Milano, 1981.

Marcuse H., L'uomo a una dimensione. L'ideologia di una società industriale avanzata, trad. di L. Gallino, Einaudi, Torino 1977.

Martinelli A., La modernizzazione, Laterza, Bari 2000.

Marx K., Il capitale, Editori Riuniti, Roma 1970.

Mauss M., Manuale di etnografia, Jaca Book, Milano 1969; 
Mauss M., Sociologia e antropologia, Newton Compton, Roma 1976;

Mauss M., I fondamenti di un'antropologia storica, Einaudi, Torino 1998;

Mauss M., Saggio sul dono, Einaudi, Torino 2002.

Negri A., Hardt M., Impero : il nuovo ordine della globalizzazione, Rizzoli, Milano 2002.

Negri A., Movimenti nell'impero : passaggi e paesaggi, R. Cortina, Milano 2006

Nocifora E., Il lavoro difficile, Maggioli, Bologna 2007.

Nocifora E., Quando il mobbing è management. Controllo, discriminazione e violenza nelle relationi lavorative, sta in “Tutela”, II, n. 3, dicembre-marzo 2008-2009, pp. 131-142.

Polanyi K., La grande trasformazione,Einaudi, Torino, 1981.

Popper K., Società aperta universo aperto, Borla, Roma 1984.

Popper K., La società aperta e i suoi nemici, Armando, Roma 1996.

Pugliese E., Rebbeggiani E., Occupazione e disoccupazione in Italia: dal dopoguerra ai giorni nostri, Il Mulino, Nologna 2004.

Renan E., Che cos'è una nazione? E altri saggi, Donzelli, Roma 1998

Reyneri E., Sociologia del mercato del lavoro, Il Mulino, Bologna 2002.

Reyneri. E., Le forme dell'occupazione, Il Mulino, Bologna 2005.

Rifkin J., La fine del lavoro. Il declino della forza lavoro glovale e l'avvento dell'era post-mercato, Baldini \& Castoldi, Milano 2001.

Sassen S., Le città nell'economia globale, Il Mulino, Bologna 1997.

Sassen S., Città Globali, UTET, Torino 1998.

Simmel G., La metropoli e la vita dello spirito, Armando Ed., 
Roma 1995.

Simmel G., Sociologia, Ed. Comunità, Milano 1989.

Sombart W., Il capitalismo moderno, UTET, Torino, 1967;

Sombart W., Lusso e capitalismo, Edizioni del Veltro, Parma 1987.

Tönnies F., Comunità e società, Ed. Comunità, Milano 1963.

Touraine A., La società post-industriale, Il Mulino, Bologna 1974, Touraine A., Critica della modernità, Il Saggiatore, Milano 1993. Veblen T., La teoria della classe agiata, Einaudi, Torino, 1971.

P. Vilar, Oro e moneta nella storia: 1450-1920, Laterza, Bari 1971.

Wallerstein I., Il sistema mondiale dell'economia moderna, Il mulino, Bologna 1986

Wallerstein I., Il declino dell'America, Feltrinelli, Milano 2004

Wallerstein I., Le scienze sociali. Come sbarazzarsene, Il Saggiatore, Milano 1995.

\section{FORMA DE CITAR ESTE TRABAJO EN BIBLIOGRAFÍAS HOW TO CITE THIS ARTICLE IN BIBLIOGRAPHIES}

Nocifora, E. (2011): "Precarietà del lavoro e crisi delle moderne forme di cittadinanza sociale", Revista Latina de Sociologia, $\mathrm{n}^{\circ} 1$ : 126-155, http://revistalatinadesociologia.com, ISSN 2253-6469 